\title{
Nanomodified Heat-Accumulating Materials with Controlled Thermophysical Characteristics
}

\author{
A.V. Shchegolkov ${ }^{* 1}$, Rama S. Verma ${ }^{2}$, Seeram Ramakrishna ${ }^{3,4}$, P.O. Borovskikh ${ }^{1}$ \\ ${ }^{1}$ Tambov State Technical University, 106, Sovetskaya St., Tambov, 392000, Russia; \\ ${ }^{2}$ Department of Biotechnology, Indian Institute of Technology, Madras, 201, Chennai 600 036, India; \\ ${ }^{3}$ Shenzhen Institutes of Advanced Technology, Chinese Academy of Sciences, 1068 Xueyuan Avenue, Shenzhen, China; \\ ${ }^{4}$ Center for Nanofibers and Nanotechnology, National University of Singapore, 9, Engineering Drive 1, Singapore \\ * Corresponding author. Tel.: + 7 (4752) 6392 93; fax: +7 (4752) 6355 22. E-mail: Energynano@yandex.ru
}

\begin{abstract}
The experimental studies of the dimensional stability of heat-accumulating nanomodified paraffin granules in the controlled electromagnetic field are carried out. The properties of the magnetic susceptibility of nanomodified paraffin, its electrical and thermal characteristics at the phase transition temperature, as well as the behavior of the modified paraffin in the liquid media (glycerin, distilled water, ethylene glycol, propylene glycol and mineral motor oil) in the controlled electromagnetic field are studied. The experimental data on the effect of various types of CNTs synthesized on $\mathrm{Ni} /{ }_{0.3} \mathrm{MgO}$; $\mathrm{Ni} /{ }_{0.5} \mathrm{MgO} ; \mathrm{Ni} / 0.16 \mathrm{MgO}$ on thermal conductivity and heat capacity of nanomodified paraffin is obtained. The effect of CNTs on the density of granulated heat-accumulating nanomodified paraffin granules and the subsequent distribution in various types of liquids has been established. The distribution of the temperature field on the surface of a liquid containing heat-accumulating nanomodified paraffin granules in the controlled magnetic field is investigated.
\end{abstract}

\section{Keywords}

Heat accumulator; carbon nanotubes; paraffin; thermal conductivity; heat capacity.

(C) A.V. Shchegolkov, Rama S. Verma, Seeram Ramakrishna, P.O. Borovskikh, 2019

\section{Introduction}

Improving the energy efficiency of the industry is associated with the development of new technical systems for the accumulation and distribution of thermal energy. A special role in the creation of energy-efficient complexes can be acquired by heat accumulators. In this case, heat accumulators can be used as independent technological units, as well as components of complex technical systems, which include solar power plants. The main part of heat accumulators is heat-accumulating materials, which define the main technological modes and design parameters of heat accumulators. At the same time, heat accumulators can show their maximum energy efficiency in periodic processes in the chemical industry. Heat accumulators can play an important role in drying, heat treatment for improving the energy efficiency, as well as in improving the energy efficiency of heating systems of buildings and structures, etc.

There is a large number of heat accumulators (organic, inorganic, and eutectic) that can be identified both in terms of melting point and latent heat of fusion. However, except melting points in the working range, most heat accumulators do not fully meet the necessary criteria. This is due to the fact that materials with the effect of thermal energy accumulation during the phase transition associated with melting have low thermal conductivity. Another problem of using phase transition materials is the tendency to ignite at high temperatures, as well as high corrosivity when using salts or crystalline hydrates. For this reason, they use available materials and try to improve their thermophysical properties to increase the efficiency of such heat materials in the composition of designed heat accumulators [1]. 
The most effective heat materials are considered to be materials capable of accumulating thermal energy in the phase transition [2-7]. Further development of the heat materials can be associated with obtaining new functionality, due to the implementation of ferromagnetic properties in the material itself. However, materials with the ability to reversible phase transitions, as a rule, are paramagnetic. To give them ferromagnetic properties, it is necessary to introduce ferromagnetic particles. This is difficult, since ferromagnetic particles act as foreign structures and precipitate. For this reason, special technological methods should be considered, which will allow introducing ferromagnetic particles into their structure.

In the field of creating heat materials, new technologies are being designed related to the development of granular heat materials [8].

The study [9] uses a geopolymer obtained from industrial waste and dune sand by alkali-activated polymerization. This material is used as a shell to prevent leakage of paraffin encapsulated in expanded clay.

However, the granulation technology significantly reduces the storage capacity of phase transition materials, as the phase transition material is replaced with the shell material. In the conducted studies, the possibility of controlling the charge/discharge processes of heat accumulators due to the realization of magnetic properties for heat materials is not considered.

In [10], a technology has been developed for efficient binding of iron oxide nanoparticles with single-wall carbon nanotubes (SWCNTs) in an aqueous solution containing the sodium dodecylbenzenesulfonate surfactant. The analysis of the experimental results shows that the alignment of magnetic-sensitive nanoparticles $\left(\mathrm{Fe}_{2} \mathrm{O}_{3}\right)$ associated with SWCNTs in a polymer matrix under a magnetic field can significantly improve the physical and mechanical properties of composites. At the same time, the authors of [10] consider materials without phase transitions.

The review of the articles $[1-10]$ shows that there are physicochemical approaches aimed at improving the properties of the heat materials, however, the main emphasis was placed on obtaining material with improved properties without imparting any functional properties allowing to set the properties of controlled charge/discharge modes.

The study of issues related to the development of new types of heat materials with a wide range of functional properties that can provide controlled charge/discharge modes is an important scientific task.

\section{Materials and methods}

\section{Paraffin modification technique by carbon nanotubes}

In order to obtain heat materials, a CNT (a separate type of CNT for each sample) was placed in a $0.002 \mathrm{~m}^{3}$ silicone container and paraffin of grade $\mathrm{P} 2$ heated to $80^{\circ} \mathrm{C}$ was added. Then the resulting melt was treated with ultrasound with a frequency of $22.5 \mathrm{kHz}$ for 1.5 hours while stirring with a mechanical stirrer $(100 \mathrm{rpm})$ and maintaining the temperature at $70{ }^{\circ} \mathrm{C}$.

To create a nanomodifier (CNT), three $\mathrm{Ni} / \mathrm{MgO}$ catalytic systems were obtained that differ in the content of the active component: $\mathrm{Ni} / 0.16 \mathrm{MgO}$; $\mathrm{Ni} / 0.3 \mathrm{MgO} ; \mathrm{Ni} / 0.5 \mathrm{MgO}$.

\section{Methods for studying the dimensional stability of heat materials in a granular state with various physical-mechanical material parameters}

\section{Investigation of the dimensional stability of granular heat materials}

Modified paraffin granules acquire a different shape during the granulation process. Granulation occurs during mechanical milling due to the translational and rotational effects on the nanomodified paraffin metal plate containing equidistant holes. The shape of the granules can vary by changing the diameter of the holes. Paraffin is the phase transition material in the granules. CNT provides shaping and improves thermal conductivity, and also reinforces paraffin to some extent. The change in the shape of the granules can be influenced by the temperature of the medium in which the granules will be located, as well as the ability of the medium to dissolve the material of the bond - the matrix of the granules (paraffin) (Fig. 1).

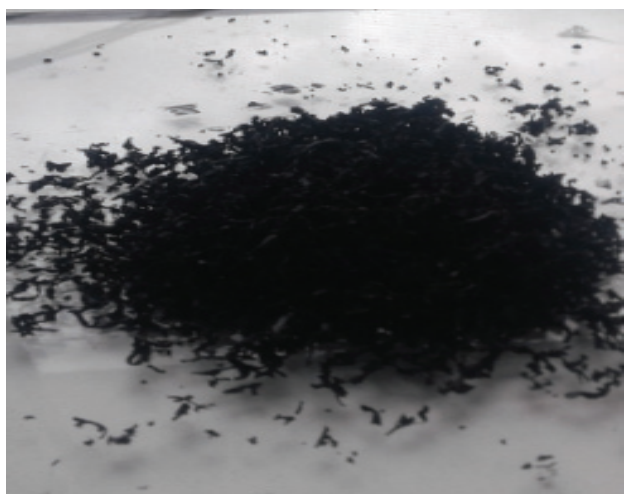

Fig. 1. Magnetically controlled granular material 
Granulation is a method of distributing a phase transition material in the medium that will ensure efficient heat transfer. Reducing the size of the granules contributes to the efficiency of heat transfer. It is necessary to achieve the greatest minimization of the granules size. To do this, a two-step approach should be considered. At the first stage the granules with the smallest possible size are produced. In the second stage, due to the dissolution of the granules in a liquid medium, the size of the granules decreases. At the same time, the granules with the minimum sizes should have constant sizes in the established temperature range. The density of the granules varies depending on the density of the liquid in which they will be distributed. This is due to the need for the uniform distribution of the granules in the liquid.

\section{Method for evaluating the dimensional stability}

To assess the dimensional stability of granulated nanomodified paraffin, four types of nanomodified material with different types of CNTs (obtained on different catalysts No. 1-4) and different weight percentages of CNT were used.

To measure the density of heat material granules from nanomodified paraffin, cylinders with a height of $1 \mathrm{~cm}$ and a radius of $4 \mathrm{~cm}$ are obtained. A loading weight (from 30 to 100 grams) is placed on the surface of the cylinder. Then the cylinder is placed in a container with water and the density of the modified paraffin is calculated from the volume of displaced water, taking into account the applied load. The granular material with an appropriate density is placed in different types of liquids (propylene glycol, ethylene glycol, mineral oil, glycerin, distilled water).

The liquids were placed in plastic cylindrical flasks (Fig. 2). The flasks were placed in water.

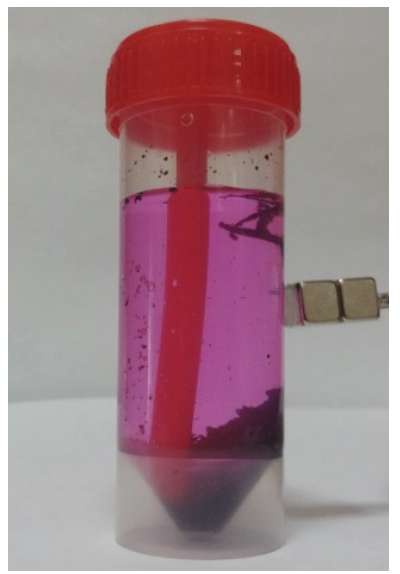

Fig. 2. Investigation of the magnetic susceptibility of the granules distributed in the liquid
The water temperature was set in the range from 25 to $95^{\circ} \mathrm{C}$. The temperature change step was $5{ }^{\circ} \mathrm{C}$. The exposure time at an appropriate temperature was 10 minutes. Different types of granules obtained on different types of CNTs were investigated.

\section{Methods for studying the magnetic susceptibility of nanomodified materials}

The study of magnetic susceptibility for magnetically controlled granules was carried out using a permanent magnet on a flask containing granules (see Fig. 2). A magnet was brought to the side of the flask - the granules were attracted to the magnet, thereby changing their position in the volume of the plastic flask.

\section{Methods for studying heat capacity and thermal conductivity of nanomodified paraffin}

Thermophysical parameters of modified paraffin were measured on the devices IT $-\lambda-400$ (measurement of thermal conductivity $\left(\mathrm{W} / \mathrm{m}{ }^{\circ} \mathrm{C}\right)$ ) and IT-C-400 (measurement of heat capacity, $\left(\mathrm{J} / \mathrm{kg}{ }^{\circ} \mathrm{C}\right)$ ) in the monotonic heating mode with an average speed of $0,2^{\circ} \mathrm{C} / \mathrm{s}$ under adiabatic conditions.

\section{Methods for studying the temperature field}

To study the temperature field distribution, a noncontact temperature measurement method was used. A FLUKE Ti9 thermal imager with a $160 \times 120$ radiation detector and a matrix in the focal plane and the measurement range from -20 to $+250{ }^{\circ} \mathrm{C}$, with an error of $\pm 5^{\circ} \mathrm{C}$, was used.

\section{Methods for studying the behavior of modified paraffin in liquid conditions}

The electromagnetic field can induce the current in nanomodified paraffin granules. This will lead to the fact that the electric current in the granules will cause heat generation. The implementation of a controlled charge/discharge mode and the transfer of the granules to the zone of least heat exchange can help to increase the efficiency of thermal energy storage. Electroconvection can be achieved using high-voltage electric fields. At the same time, using the approach associated with the formation of electrical conductivity directly in a liquid due to CNTs and using a magnetic field from a permanent magnet, it is possible to provide controlled fluid rotation. Thus, the parameters of the fluid rotation will affect the characteristics of the fluid associated with the conductivity. To implement 
electroconvection, distilled water, in which CNTs are distributed, is most suitable. After the CNT distribution, nanomodified paraffin granules are introduced. Then the water together with the granules is heated to a temperature of $70{ }^{\circ} \mathrm{C}$ and affected by ultrasound. Two copper electrodes with a cross section of $3 \mathrm{~mm}$ are placed in the container. One electrode in the form of a ring is placed on the bottom of the container, and the other in the form of a rod is installed in the middle of the container. A constant voltage in the range from 2 to $40 \mathrm{~V}$ is applied to the electrodes.

\section{Results and discussion}

After the CNT paraffin is modified, a black, opaque material is obtained (Fig. 3).

All catalysts containing more than $50 \%$ of the active component have a coral-like structure (Fig. 4a). When decreasing its content in the catalyst composition, a change in their structure occurs (Fig. 4b).

These catalysts were used for the synthesis of carbon nanostructured materials (CNM) using the method of chemical vapor deposition. Characteristics of the obtained catalysts are presented in Table 1 .

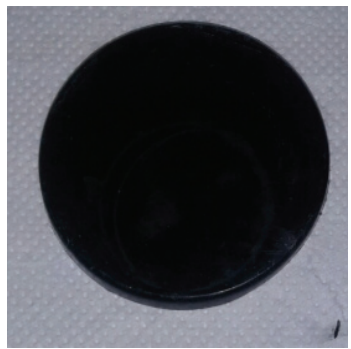

Fig. 3. Paraffin modified by CNT

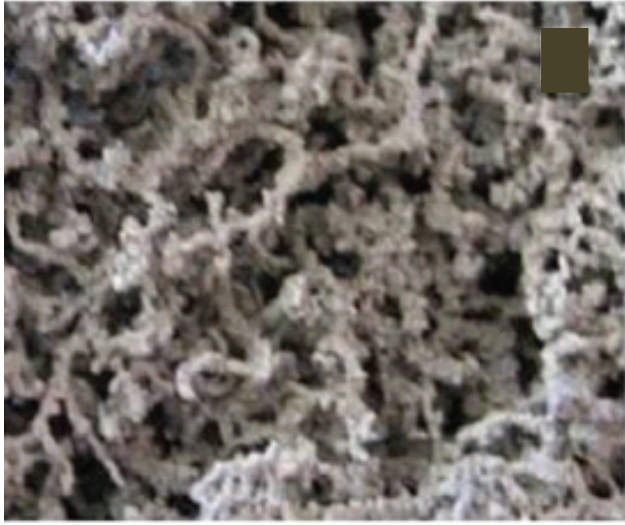

a)
The synthesized CNT was investigated using scanning (SEM) and transmission (TEM) electron microscopy. The morphology of CNM synthesized on the obtained catalysts is shown in Fig. 5.

The analysis of micro images brings us to the conclusion that all CNM samples consist of filamentary structures. The structures synthesized on $\mathrm{Ni} / 0.3 \mathrm{MgO}$ и $\mathrm{Ni} / 0.16 \mathrm{MgO}$ catalysts have a clearer morphology. The diameter of carbon filamentary structures synthesized on $\mathrm{Ni} / 0.16 \mathrm{MgO}$ и $\mathrm{Ni} / 0.3 \mathrm{MgO}$ catalysts has a diameter of $\sim 30-60 \mathrm{~nm}$.

The comparison of the results obtained in this method with the tabular data of the reference book gives an error of no more than $1 \%$.

The introduction of CNT in the paraffin matrix leads to the decrease in its density (Table $2-4$ ). The lowest density is characteristic of sample No. 4. The initial paraffin density is $890 \mathrm{~kg} / \mathrm{m}^{3}$. The decrease in the density of paraffin will lead to the fact that the granules float to the surface of the liquid. To stabilize the density of the granular material and enhance the magnetic properties, it is necessary to introduce

Table 1

\section{Characteristics of $\mathrm{Ni} / \mathrm{MgO}$ catalysts}

\begin{tabular}{ccc}
\hline $\begin{array}{c}\text { Catalyst } \\
\text { composition }\end{array}$ & $\begin{array}{c}\text { Specific surface, } \\
\mathrm{m}^{2} / \mathrm{g}\end{array}$ & $\begin{array}{c}\text { Effeciency, } \\
G_{\text {carb }} / G_{\text {kat }}\end{array}$ \\
\hline $9: 1$ & 51.9 & 9.64 \\
$8: 2$ & 55.6 & 11.30 \\
$7: 3$ & 60.8 & 4.90 \\
\hline
\end{tabular}

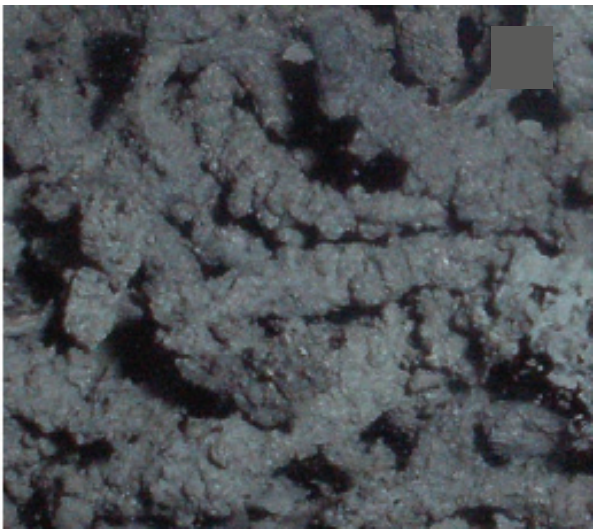

b)

Fig. 4. Structure of the $\mathrm{Ni} / \mathrm{MgO}$ catalyst:

$a$ - content of the active component more than $50 \% ; b$ - content of the active component less than $50 \%$ 


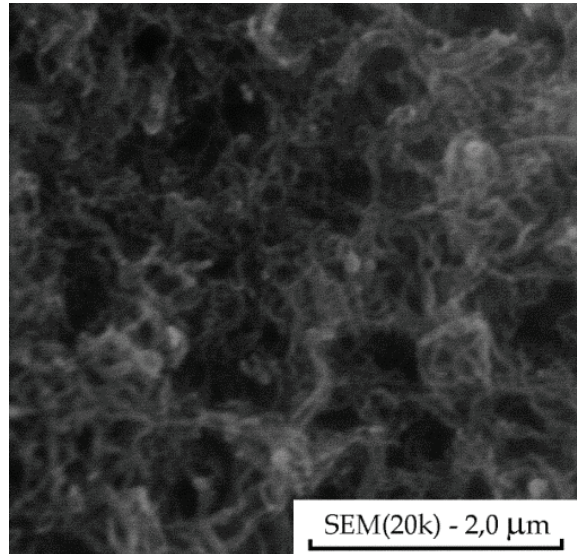

a)

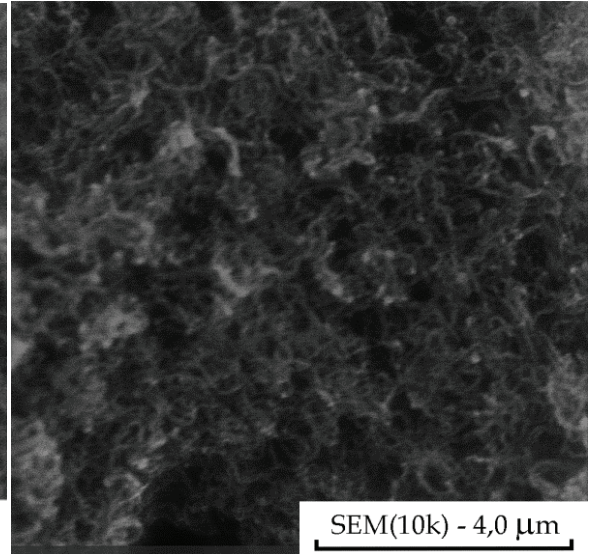

b)

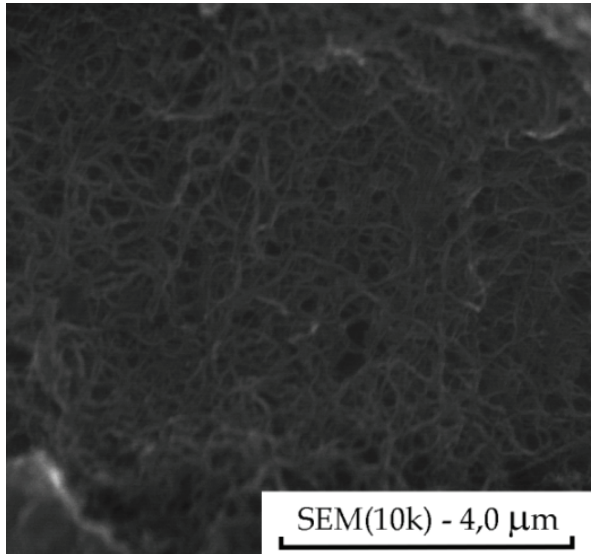

c)

Fig. 5. Morphology of CNTs synthesized on the catalyst: $a-\mathrm{Ni} / 0.3 \mathrm{MgO} ; b-\mathrm{Ni} / 0.5 \mathrm{MgO} ; c-\mathrm{Ni} / 0.16 \mathrm{MgO}$

Table 2

Paraffin modified by CNT on the catalyst No. 1

\begin{tabular}{cccc}
\hline No. & CNT type & CNT concentration, \% & $\begin{array}{c}\text { Density, } \\
\mathrm{kg} / \mathrm{m}^{3}\end{array}$ \\
\hline 1 & 4 & 860 \\
2 & CNT No.1 & 8 & 830 \\
3 & & 12 & 820 \\
4 & 16 & 800 \\
\hline
\end{tabular}

Table 3

Paraffin modified by CNT on the catalyst No. 2

\begin{tabular}{cccc}
\hline No. & CNT type & CNT concentration, \% & $\begin{array}{c}\text { Density, } \\
\mathrm{kg} / \mathrm{m}^{3}\end{array}$ \\
\hline 1 & 4 & 855 \\
2 & CNT No. 2 & 8 & 825 \\
3 & & 12 & 815 \\
4 & 16 & 795 \\
\hline
\end{tabular}

Table 4

Paraffin modified by CNT on the catalyst No. 3

\begin{tabular}{cccc}
\hline No. & CNT type & CNT concentration, \% & $\begin{array}{c}\text { Density, } \\
\mathrm{kg} / \mathrm{m}^{3}\end{array}$ \\
\hline 1 & 4 & 854 \\
2 & CNT No. 3 & 8 & 824.5 \\
3 & & 12 & 814 \\
4 & 16 & 878 \\
\hline
\end{tabular}

a ferromagnetic powder. Additives of ferromagnetic powder will be from 10 to $30 \%$ mass. In this case, the density will increase. Taking into account the density of the liquid, it is possible to choose the density of nanomodified paraffin. This will ensure the granules soar in the liquid volume, which will contribute to an even accumulation of thermal energy. For glycerin, the density of nanomodified paraffin will be $1260 \mathrm{~kg} / \mathrm{m}^{3}$, for ethylene glycol - $999 \mathrm{~kg} / \mathrm{m}^{3}$, for propylene glycol $1036 \mathrm{~kg} / \mathrm{m}^{3}$, for mineral oil $-995 \mathrm{~kg} / \mathrm{m}^{3}$, for distilled water $-1000 \mathrm{~kg} / \mathrm{m}^{3}$.

In the range of up to $95^{\circ} \mathrm{C}$, all the granules (synthesized by CNT on 4 types of catalysts), located in various liquids, retained their initial shape and did not form agglomerates. However, the granules in the mineral oil dissolve to smaller granules in diameter (Fig. 6). With the correctly chosen density, the granules show the maximum possible stability in the flask volume and show no shrinkage. On CNTs synthesized on catalysts No. 1, 2 and 3, which modify paraffin, there is a loss of shape and assembly into large aggregates, as well as complete melting at temperatures of about $90^{\circ} \mathrm{C}$.

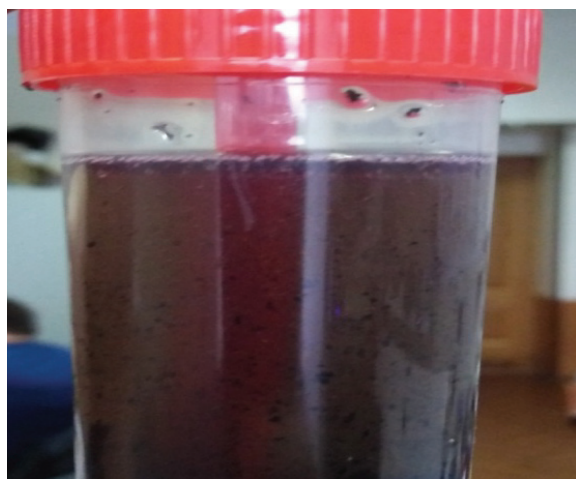

Fig. 6. Granular material in mineral oil 


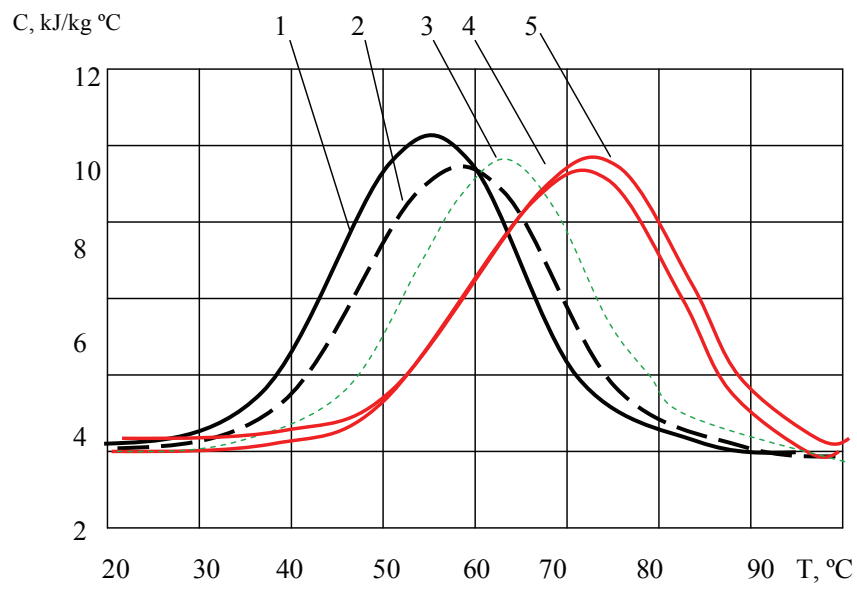

a)

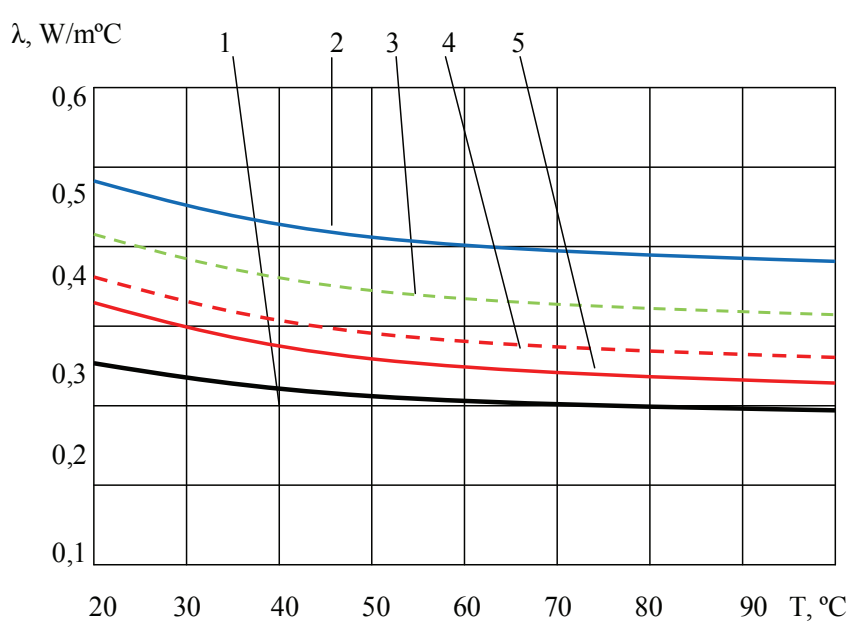

b)

Fig. 7. Temperature dependency of the heat capacity $(a)$ and thermal conductivity $(b)$ of paraffin modified by CNT: 1 - paraffin; 2 - paraffin modified by CNT with catalyst No. 1;3 - paraffin modified by CNT with catalyst No. 2; 4 - paraffin modified by CNT with catalyst No. $3 ; 5$ - paraffin modified by CNT with catalyst No. 4

The best results in dimensional stability are shown by samples of modified CNTs synthesized on the 4th catalyst and correspond to the maximum possible CNT concentration in paraffin. The influence of the magnetic field on the movement of the granules in the liquid was established. The mode of the granules movement is already observed when the value of the magnetic induction is $100 \mathrm{mT}$.

According to experimental studies, the temperature dependencies of the heat capacity for pure paraffin and nanomodified paraffins with different types of catalysts No. 1, 2, 3 and 4 were established (Fig. 7a).
Fig. $7 b$ presents the study of the temperature dependency of the thermal conductivity of paraffin modified by CNT.

\section{Electrophysical parameters of nanomodified paraffin}

Fig. 8 presents the distribution of equipotential lines on the surface of nanomodified paraffin.

The conducted research shows (see Fig. 8) that an electric current can be induced in the conditions of the electromagnetic field in nanomodified paraffin.

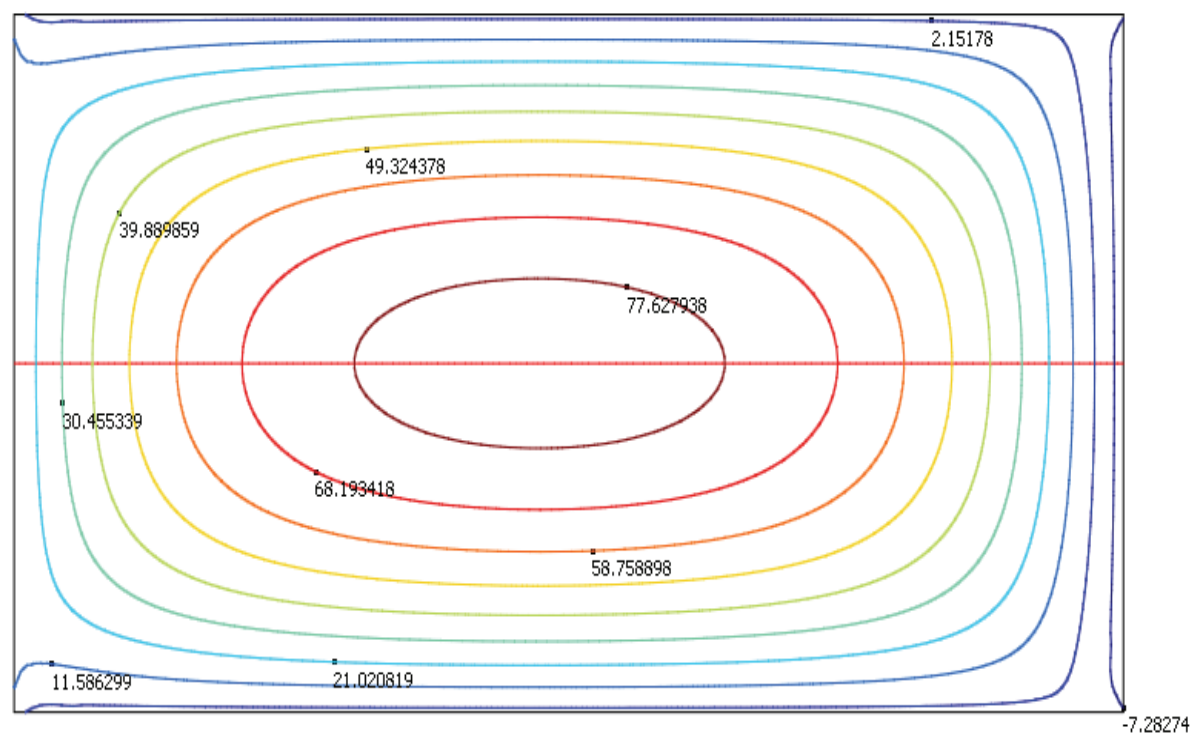

$\varphi, \mathrm{V}$

Fig. 8. Distribution of equipotential lines on the surface of nanomodified paraffin (longitudinal arrangement of electrodes) 


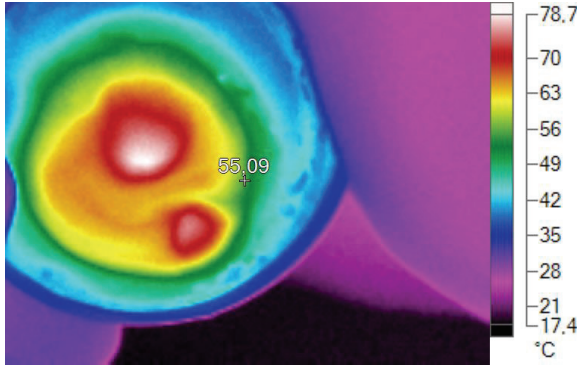

a)

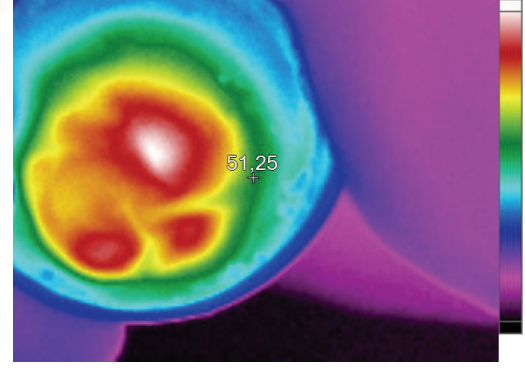

b)

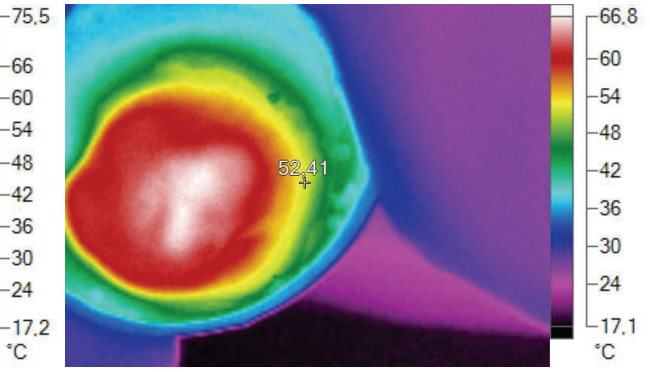

c)

Fig. 9. Thermogram of liquid surface with granulated nanomodified paraffin

\section{Investigation of the temperature field distribution in a fluid containing magnetically controlled heat material}

When a permanent magnet moves in the lower part of a container with the granulated heat material, the liquid rotates in the volume due to the movement of the granules.

Fig. 9 show the change in the temperature field under the action of a magnetic field.

The analysis of Fig. 9 shows that under the action of a magnetic field in the heat material volume, the temperature field is redistributed. Due to this effect, it is possible to remove stagnant zones during the heat exchange during the charge/discharge process. Nanomodified paraffin provides accumulation of heat, while the liquid intensifies internal heat exchange between individual granules. Controllability of the internal structure allows to intensify the charge/discharge process.

\section{Conclusion}

The studies have shown the ability to control charge/discharge modes in the heat-accumulating material. To create magnetically controlled granules, CNTs synthesized on catalysts were used: $\mathrm{Ni} / 0.3 \mathrm{MgO}$; $\mathrm{Ni} / 0.5 \mathrm{MgO} ; \quad \mathrm{Ni} / 0.16 \mathrm{MgO}$. The behavior of the magnetically controlled material in various types of liquids, which can be used as heat carriers or accumulators of thermal energy, was investigated. Granular heat-accumulating material has a wide range of functional properties that provide the possibility of thermal energy accumulation both in direct contact with the coolant and in non-contact indication with the help of the electromagnetic field. The granulated heataccumulating material can be used in solar energy technologies - in oil tanks, accumulators and chemical industry technologies for effective temperature control or thermal regulation.

\section{Acknowledgments}

The study was supported by the Russian Foundation for Basic Research and the Administration of Education and Science of the Tambov Region within the framework of the research project No. 18-43-680012.

\section{References}

1. Sharma S., Sagara K. Latent Heat Storage Materials and Systems. International Journal of Green Energy, 2005, issue 2, pp. 1-56. doi: 10.1081/GE-200051299

2. Zhao C. Heat Transfer Enhancement for Thermal Energy Storage Using Metal Foams Embedded within Phase Change Materials (PCMs). Solar Energy, 2010, vol. 84, pp. 1402-1412. doi: 10.1016/J.SOLENER.2010.04.022

3. Fan L. Thermal Conductivity Enhancement of Phase Change Materials for Thermal Energy Storage: a Review. Renewable and sustainable energy reviews, 2011, vol. 15. pp. 24-46. doi: 10.1016/j.rser.2010.08.007

4. Warzoha R., Zhang D., Feng G., Fleischer A. Engineering Interfaces in Carbon Nanostructured Mats for the Creation of Energy Efficient Thermal Interface Materials. CARBON, 2013, vol. 61. pp. 441-457. doi: 10.1016/j.carbon.2013.05.028

5. Warzoha R. Effect of carbon nanotube interfacial geometry on Thermal Transport in Solid-Liquid Phase Change Materials. Applied Energy, 2015, vol. 154, pp. 271-276. doi: 10.1016/ j.apenergy.2015.04.121

6. Li M. Carbon Nanotube/Paraffin/Montmorillonite Composite Phase Change Material for Thermal Energy Storage. Solar energy, 2017, vol. 146, pp. 1-7. doi: 10.1016/j.solener. 2017.02.003

7. Zhang N. Effect of Carbon Nanotubes on the Thermal Behavior of Palmitic-Stearic Acid Eutectic Mixtures as Phase Change Materials for Energy Storage. Solar Energy, 2014, vol. 110, pp. 64-70. doi: 10.1016/j.solener.2014.09.003

8. Jayalath A., Nicolas R.S., Sofi M., Shanks R., Ngo T., Aye L., Mendis P. Properties of Cementitious Mortar and Concrete Containing Micro-encapsulated Phase Change Materials. Constr. Build. Mater., 2016, vol. 120, pp. 408-417. doi: 10.1016/j.conbuildmat.2016.05.116

9. Xuesen L., Guo H., Cui X. Preparation of Paraffin-Based Phase-Change Microcapsules and Application in Geopolymer Coating. Journal of Coatings Technology and Research, 2018, vol. 15, issue 4, pp. 867-874. doi: 10.1007/s11998-018-0071-6

10. Mingrui L., Hammad A., Hammad A., Haiping H., Peterson G. Polymer Nanocomposites with Improved Mechanical and Thermal properties by Magnetically Aligned Carbon Nanotubes. Polymer, 2019, vol. 166, pp. 81-87. doi: 10.1016/j.polymer.2019.01.031 